\title{
Variation de la sensibilité au sel en fonction du stade de développement chez la lentille (Lens culinaris $L$ )
}

\author{
M Lachaâl ${ }^{1 *}, \mathrm{C}$ Abdelly ${ }^{1}, \mathrm{C}$ Grignon ${ }^{2}$, A Soltani ${ }^{1}, \mathrm{M}$ Hajji ${ }^{3}$ \\ 1 Unité de biotechnologie végétale et de ressources génétiques, nutrition minérale, INRST, BP 95, 2050 Hammam Lif, Tunisie; \\ 2 Biochimie et physiologie moléculaire des plantes (CNRS Ura 2133), Ensa-Inra, F-34060 Montpellier cedex 1, France; \\ 3 Physiologie végétale, faculté des sciences de Tunis, campus universitaire, 1060 Tunis, Tunisie
}

(Reçu le 29 décembre 1995 ; accepté le 2 mai 1995)

\begin{abstract}
Résumé - Dans le but de comparer la sensibilité au sel de la lentille, Lens culinaris L, à deux stades de développement (premier mois de développement végétatif, et stade floraison), deux séries d'expériences sont réalisées sur quatre populations locales provenant de diverses régions de la Tunisie. Dans la première expérience, les plantes sont cultivées sous plafond lumineux, sur milieu liquide dépourvu de $\mathrm{NaCl}$ pendant 3 semaines. Le milieu est ensuite additionné de $\mathrm{NaCl} 50,100$ ou $150 \mathrm{mM}$, et les plantes sont récoltées 9 jours plus tard. Dans la seconde expérience, les cultures sont conduites sur sable inerte en pots. Pendant les 3 premières semaines, toutes les plantes sont irriguées avec une solution nutritive contenant $\mathrm{NaCl} 10 \mathrm{mM}$ (concentration dans l'eau de ville). La concentration de $\mathrm{NaCl}$ est ensuite portée à $36 \mathrm{mM}$ pour la moitié des plantes. La récolte est faite 2 mois plus tard, les plantes étant alors âgées de 11 semaines, et ayant atteint le stade floraison depuis 2 semaines. Les plantes aux stades juvéniles ( 1 mois) mises en présence de $\mathrm{NaCl} 50 \mathrm{mM}$ ne présentent aucun ralentissement de croissance (première expérience). Des inhibitions de croissance, différentes entre les populations, apparaissent aux stades plus tardifs, quand les plantes sont traitées par $\mathrm{NaCl} 36 \mathrm{mM}$ (deuxième expérience). À ces stades, un dessèchement d'une partie des feuilles intervient, qui paraît lié à une accumulation excessive de $\mathrm{Na}^{+}$et $\mathrm{Cl}^{-}$. Les différences de sensibilité au sel des populations sont liées à des différences de vitesse de production des feuilles. Les populations à croissance rapide, qui mettent en place de nouvelles feuilles plus vite que l'accumulation de $\mathrm{Na}^{+}$et $\mathrm{Cl}^{-}$n'élimine les anciennes, sont les plus résistantes au sel.
\end{abstract}

lentille / sensibilité à $\mathrm{NaCl}$ / vitesse de croissance / accumulation d'ions / stade de développement

Summary - Variation in salt sensitivity during development of lentils. The sensitivity of Lens culinaris $\mathrm{L}$ to $\mathrm{NaCl}$ was compared at two developmental stages. Four local populations of lentil taken from various regions of Tunisia were used. Seedlings were grown for 3 weeks on liquid medium, under artificial light. The medium was then supplemented with $\mathrm{NaCl}(50,100$ and $150 \mathrm{mM})$. The plants were harvested 9 days later. In a second experiment, the plants were grown for 3 weeks on sand irrigated with a medium containing $10 \mathrm{mM} \mathrm{NaCl}$. For half of them, the $\mathrm{NaCl}$ concentration was then increased to $36 \mathrm{mM}$. The experiment ended when the plants were 11 week old (2 weeks after the beginning of flowering). In young plants (1-month-old) treated with $50 \mathrm{mM} \mathrm{NaCl}$, no growth inhibition was observed. Growth inhibitions, variable according to populations, appeared at late developmental stages in plants treated with $36 \mathrm{mM} \mathrm{NaCl}$. Excess accumulation of $\mathrm{Na}^{+}$and $\mathrm{Cr}$, associated with leaf dehydratation, occurred in leaves. The differential effect of $\mathrm{NaCl}$ on growth between populations was related to differences in the rate of leaf production. In populations with high growth rate, the production of new leaves was more rapid than the elimination of old leaves by $\mathrm{Na}^{+}$and $\mathrm{Cl}^{-}$excess accumulation. These populations were more tolerant to $\mathrm{NaCl}$.

lentil / sensitivity of $\mathrm{NaCl}$ / growth rate / ion accumulation / development stage

* Correspondance et tirés à part.

Abréviations : MF : matière fraîche; MS : matière sèche ; CMR : croissance moyenne relative 


\section{INTRODUCTION}

Une variabilité intraspécifique pour la tolérance à la salinité a été repérée chez beaucoup d'espèces (Mass et Hoffman, 1977 ; Epstein et al, 1980 ; Greenway et Munns, 1980 ; Epstein, 1983 ; Ashraf et Waheed, 1990). Pour des raisons de commodité, de nombreuses explorations de cette variabilité ont été conduites sur des plantes très jeunes, à des stades végétatifs. Cette approche est justifiée par le fait que la réponse des plantules est parfois fortement prédictive de celle des plantes adultes (Greenway, 1965 ; Blum, 1985). Chez le maïs, par exemple, les effets du sel (en \% de réduction par rapport au témoin) sont les mêmes pour la croissance pendant le premier mois et pour la production en grain (Katerji et al, 1994). Les vitesses d'émergence et de croissance végétative des plantules ont été mentionnées comme des indicateurs fiables de la tolérance pour classer lignées et cultivars (Shannon et al, 1983 ; Lehman et al, 1984 ; Ashraf et al, 1986a, b). Une classification de plusieurs lignées de lentille, basée sur des mesures de la germination et de la croissance sur milieu salé, a été confirmée pour les lignées tolérantes par une étude au champ (Jana et Slinkard, 1979). Mais, en dépit de ces éléments encourageants, la pertinence des critères précoces pour prédire le rendement agronomique, ou même simplement le développement végétatif de la plante adulte soumise à la contrainte saline ne peut être admise a priori. Dans un travail d'exploration réalisé sur 133 accessions de lentille, Ashraf et Waheed (1990) ont montré qu'il n'y a pas de relation claire entre la tolérance au sel estimée au stade germination et celle au stade plantule. En outre, dans d'autres exemples, les réponses à la salinité sont différentes, ou même opposées, entre les stades juvéniles et adultes (Abel et Mackenzie, 1964 ; Greenway, 1965 ; Chapman, 1968 ; El Gibaly et Goumah, 1969 ; Ream et Furr, 1976 ; Norlyn, 1980 ; Norlyn et Epstein, 1984 ; Kuiper et al, 1988 ; Zid, 1989). L'écart entre la tolérance au stade plantule et celle au stade adulte (Shannon, 1979 ; Kingsbury et Epstein, 1984) peut traduire des différences entre les mécanismes impliqués d'un stade de développement à un autre (Hunt, 1965).

En Tunisie, les sols affectés par la salinité naturelle couvrent 1,5 million d'hectares, soit à peu près $25 \%$ de la surface totale des sols cultivables du pays. D'autre part, le développement des cultures irriguées avec des eaux de mauvaise qualité titrant jusqu'à 5 g.L $\mathrm{L}^{-1}$ de sels (Hachicha et al, 1994) fait que le quart environ des superficies irriguées est affecté par le sel. Notre travail d'exploration de la variabilité de la tolérance au sel chez la lentille entre dans le cadre de programmes d'amélioration de la productivité végétale sur ces sols par le tri de populations locales tolérantes. Dans cette étude, nous comparons les effets de $\mathrm{NaCl}$ sur l'installation du couvert chez des plantules âgées de 3 semaines en culture hydroponique et en pot, et chez des plantes adultes en pot, pour identifier les raisons des différences des résultats des deux démarches. L'étude porte sur quatre populations locales de lentille provenant de différentes régions de la Tunisie.

\section{MATÉRIEL ET MÉTHODES}

Les semences de Lens culinaris $L$ nous ont été fournies par le laboratoire de génétique des légumineuses alimentaires de l'Institut national de recherche agronomique tunisien. Les populations sont désignées par les abréviations des noms des localités où elles sont cultivées. Elles ont été triées selon certains critères tels que la forme et la couleur des graines, la précocité et la productivité ou le rendement. Les populations Ma1, Wes et Com sont à graines larges et vertes et Tam, variété naine et précoce, porte des graines petites et rouges. Les premières, à cotylédons jaunes, appartiennent au groupe des Macrosperma et la dernière à celui des Microsperma, à cotylédons orangés. La ramification est la même dans les deux groupes (environ 30 rameaux par plante à l'âge de 9 semaines)

Les deux expériences sont faites, l'une sur des plantes cultivées en milieu liquide (durée de la culture : 1 mois), et l'autre sur des plantes cultivées dans des pots remplis de sable inerte (durée : 11 semaines). Dans la première expérience, 160 plantes sont utilisées. Après germination à $25^{\circ} \mathrm{C}$, les plantules sont repiquées sur de l'eau distillée (160 individus), dans des conditions contrôlées (photopériode : 12 heures, rayonnement efficace $150 \mu \mathrm{mol} . \mathrm{m}^{-2} . \mathrm{s}^{-1}$; température et humidité relative : respectivement $30{ }^{\circ} \mathrm{C}$ et $60 \%$ le jour, $20{ }^{\circ} \mathrm{C}$ et $80 \%$ la nuit). Dix jours après le semis, l'eau est remplacée par une solution nutritive d'abord diluée deux fois, puis complète (tableau I). Au terme de la troisième semaine (stade de 6 à 7 feuilles), des plantes sont transférées sur la solution nutritive additionnée de $\mathrm{NaCl} 50,100$ ou $150 \mathrm{mM}$, d'autres plantes restant sur la même solution. Chaque traitement comporte huit plantes. Les doses de $\mathrm{NaCl}$, à l'exception de la plus forte, sont proches de celles des eaux d'irrigation qui peuvent atteindre jusqu'à $5 \mathrm{~g} . \mathrm{L}^{-1}$ au cours des périodes de sécheresse. Deux récoltes sont faites, l'une au moment de l'application du sel, et l'autre 9 jours après. Les racines, les tiges et les feuilles sont séparées, pesées puis séchées à $80^{\circ} \mathrm{C}$ en vue d'être analysées. Pendant la culture, les milieux sont renouvelés deux fois par semaine et les solutions sont aérées à l'aide de capillaires de plastique, reliés à des pompes d'aquarium. 
Dans la deuxième expérience les cultures sont conduites dans une serre vitrée, sous éclairement solaire, en pots d'argile (capacité 2,7 kg) remplis de sable inerte lavé à l'acide chlorhydrique et rincé abondamment à l'eau. Les valeurs de la température au midi solaire sont comprises entre $22{ }^{\circ} \mathrm{C}$ et $33^{\circ} \mathrm{C}$, et celles de l'humidité relative entre 40 et $60 \%$ (respectivement $10^{\circ} \mathrm{C}-15^{\circ} \mathrm{C}$, et $75-95 \%$ à la fin de la nuit). Les plantules sont obtenues par semis directement dans les pots de culture (deux par pot, densité équivalente à 30 plantes par mètre carré). Les pots sont irrigués avec le milieu de base (tableau I) dilué cinq fois dans une eau de ville contenant $\mathrm{NaCl} 10 \mathrm{mM}$. Au stade six à huit feuilles, soit 3 semaines après le semis (mi-février), 60 plantes de chaque population sont réparties en deux lots jusqu'à la récolte finale. L'un reste irrigué avec la solution précédente, l'autre est irrigué avec cette solution additionnée de $\mathrm{NaCl}$ (concentration finale $36 \mathrm{mM}$ ) (tableau II). Des essais préliminaires ont montré que la culture peut être conduite jusqu'à la floraison si la concentration de $\mathrm{NaCl}$ ne dépasse pas cette valeur. La croissance (masse de MF et de MS), l'accumulation des ions et le nombre de feuilles saines et desséchées sont suivis de mi-février à miavril, jusqu'à la floraison ou, dans certains cas, la mort des plantes. Les comptages sont faits toutes les semaines et les récoltes tous les 15 jours. Les plantes sont âgées de 5 semaines et portent quatre ou cinq rameaux, avec cinq ou six étages foliaires au premier prélèvement. Elles sont âgées de 11 semaines au dernier prélèvement. La floraison commence à l'âge de 9 (Tam) à 10 semaines (Com, Mat et Wes). À la récolte, six plantes par traitement, provenant de trois pots, sont prélevées et séparées individuellement en racines, tiges, feuilles supérieures (les deux ou trois étages représentant la touffe apicale), feuilles inférieures (avec deux à quatre folioles seulement), et feuilles médianes (jusqu'à 14 folioles), qui occupent une position intermédiaire sur chaque rameau. Les feuilles nécrosées et desséchées sont séparées des feuilles saines dès l'apparition des nécroses.

Les ions $\mathrm{K}+, \mathrm{Na}^{+}$et $\mathrm{Ca}^{2+}$ sont dosés par émission de flamme (photomètre Eppendorf), et $\mathrm{Mg}^{2+}$ par absorption atomique (IL151, et Philips PU 9100). Les chlorures sont dosés par coulométrie à l'aide d'un chloridomètre digital (Haake Buchler). Ces dosages sont faits sur le produit d'une extraction nitrique $\left(\mathrm{HNO}_{3} \mathrm{O}, 5 \%\right)$, directement ou après réduction de la matière sèche en poudre fine à l'aide d'un broyeur à billes, type Dangoumeau.

La croissance est appréciée par le paramètre CMR (vitesse de croissance moyenne relative). La CMR est, pour un intervalle de temps $\Delta t$, la quantité de biomasse produite à partir de $1 \mathrm{~g}$ de biomasse de plante. Elle est évaluée par $\Delta \mathrm{MS} / \Delta t^{\star} \mathrm{MS}$, où $\Delta \mathrm{MS}$ est l'incrément de la matière sèche par plante pendant l'intervalle de temps $\Delta t$, et MS est la quantité moyenne de matière sèche par plante sur cet intervalle. Les moyennes et les écarts types des CMR sont calculées par la méthode proposée par Venus et Causton (1979), de manière à éviter l'appariement arbitraire des individus entre le prélèvement initial et le prélèvement final.

\section{RÉSULTATS}

\section{Effet du sel sur les jeunes plantes (culture en milieu liquide et culture en pots)}

Pendant les 9 jours de traitement avec $\mathrm{NaCl}$ $50 \mathrm{mM}$ en milieu liquide, les jeunes plantes

Tableau I. Composition de la solution nutritive (concentrations en mM).

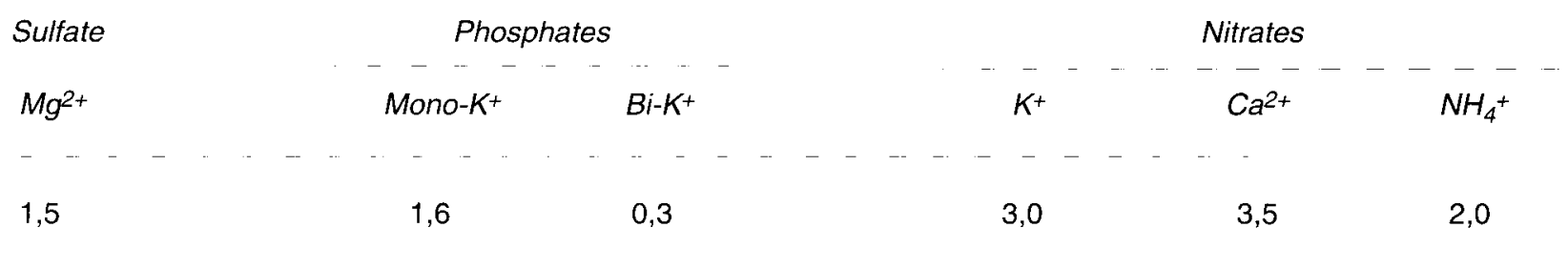

Les concentrations des oligoéléments sont (ppm) : Fe : $3 ; \mathrm{Mn}: 0,5 ; \mathrm{Cu}: 0,04 ; \mathrm{Zn}: 0,05 ; \mathrm{B}: 0,5 ;$ Mo : 0,02. Solution dite de Long Ashton (Hewitt, 1966).

Tableau II. Caractéristiques des solutions d'irrigation.

\begin{tabular}{|c|c|c|c|c|c|c|c|c|c|c|c|}
\hline Solutions & $p H$ & $\alpha E$ & $K^{+}$ & $\mathrm{Na}^{+}$ & $\mathrm{Ca}^{2+}$ & $M g^{2+}$ & $\mathrm{NH}_{4}^{+}$ & $\mathrm{NO}_{3}^{-}$ & Ct & $\mathrm{PO}_{4} \mathrm{H}_{2}^{-}$ & $\mathrm{SO}_{4}{ }^{2-}$ \\
\hline$--\quad-$ & & & & & & & & & .. - - & - & - \\
\hline $\mathrm{NaCl} 10 \mathrm{mM}$ & 7,4 & 2,2 & 1,2 & 10,4 & 4,3 & 1,8 & 0,5 & 3,0 & 10,2 & 0,4 & 2,0 \\
\hline $\mathrm{NaCl} 6 \mathrm{mM}$ & 7,4 & 5,0 & 1,2 & 35,6 & 4,3 & 1,8 & 0,5 & 3,0 & 35,8 & 0,4 & 2,0 \\
\hline
\end{tabular}


(âgées de 30 jours à la récolte finale) ne présentent pas de variation significative de la CMR par rapport aux témoins sans $\mathrm{NaCl}$. II faut utiliser une concentration de $150 \mathrm{mM}$ pour observer une baisse significative de la CMR chez Wes, Tam et Com (fig 1). Les quatre populations présentent de ce point de vue des comportements voisins. De même, les CMR des plantes en pots, âgées de 5 semaines, ne diffèrent pas significativement entre les traitements $\mathrm{NaCl} 10 \mathrm{mM}$ et $36 \mathrm{mM}$ (figs 2 et 3 ) : récolte du 2 mars.

À l'âge de 4 à 5 semaines, les plantes jeunes cultivées en présence de $\mathrm{NaCl} 50 \mathrm{mM}$ (fig 4) ou $36 \mathrm{mM}$ (fig 5) accumulent dans leurs feuilles des quantités de $\mathrm{Na}^{+}$de l'ordre de $1 \mathrm{mmol} . \mathrm{g}^{-1} \mathrm{MS}$. Les quatre populations présentent des comportements voisins. Le niveau d'accumulation de $\mathrm{Na}^{+}$ augmente avec la concentration de $\mathrm{NaCl}$ dans le milieu (fig 4), et peut alors dépasser $2 \mathrm{mmol}^{-\mathrm{g}^{-1}}$ MS. Dans ce cas, on observe un dessèchement des feuilles.

\section{Effet du sel sur les plantes adultes (cultures en pots)}

Dans le cas des plantes cultivées en présence de $\mathrm{NaCl} 10 \mathrm{mM}$, la croissance évolue de la même manière pour les quatre populations, si bien qu'au terme de l'expérience les biomasses des plantes entières sont toutes comprises entre 4 et $5 \mathrm{~g}$ MS par plante. La croissance des plantes cultivées sur le milieu contenant $\mathrm{NaCl}$ $36 \mathrm{mM}$ est fortement ralentie, en particulier chez Wes qui montre la diminution la plus marquée par rapport au traitement à faible salinité (fig 2). L'effet inhibiteur du sel sur la CMR (fig 3 ) est plus précoce chez Wes, la population la plus sensible, et à un degré moindre chez Com que chez Tam et Mat. La comparaison de l'évolution de la CMR et de la production de feuilles ( $\triangle M S$ ) explique les différences entre les populations (fig 3). La CMR est la vitesse de croissance moyenne relative de la plante entière. $\Delta \mathrm{MS}$ est l'augmentation de la masse de matière sèche des feuilles pendant les intervalles de 15 jours séparant les prélèvements pour la détermination de la CMR. Sur la figure 3, les valeurs positives de $\triangle M S$ correspondent aux masses totales de feuilles produites et les valeurs négatives aux feuilles desséchées au cours de chacun des intervalles de temps. Chez la plus résistante (Tam), une production de biomasse de nouvelles feuilles est maintenue en présence de sel, tandis qu'elle est fortement réprimée chez les plus sensibles (Mat, Wes). De même, le dessèchement qui atteint d'abord les feuilles les plus âgées (médianes et inférieures), entraîne une perte de feuilles beaucoup plus accentuée chez ces dernières populations (fig 3), de telle sorte que le bilan entre la production et le dessèchement de ces organes est fortement négatif, alors qu'il reste positif chez Tam et ne devient négatif qu'en fin d'expérience chez Com.

L'évolution du nombre de feuilles par plante au cours de la période expérimentale est illustrée sur la figure 6. Le dénombrement porte sur 30 individus par traitement et par population au début de l'expérience, et sur 12 à la fin. Les quatre populations de lentille produisent presque autant de feuilles les unes que les autres, et le nombre de feuilles par plante se situe aux alentours de 150 à 160 en fin d'expérience. Ceci permet de dire que la formation de nouvelles feuilles est peu dépendante de la présence de $\mathrm{NaCl}$, au contraire de la production de biomasse de ces organes. Le sel ne provoque qu'un léger retard dans la formation des feuilles. Cependant, il diminue leur masse individuelle (tableau III), et finit par entrainer leur

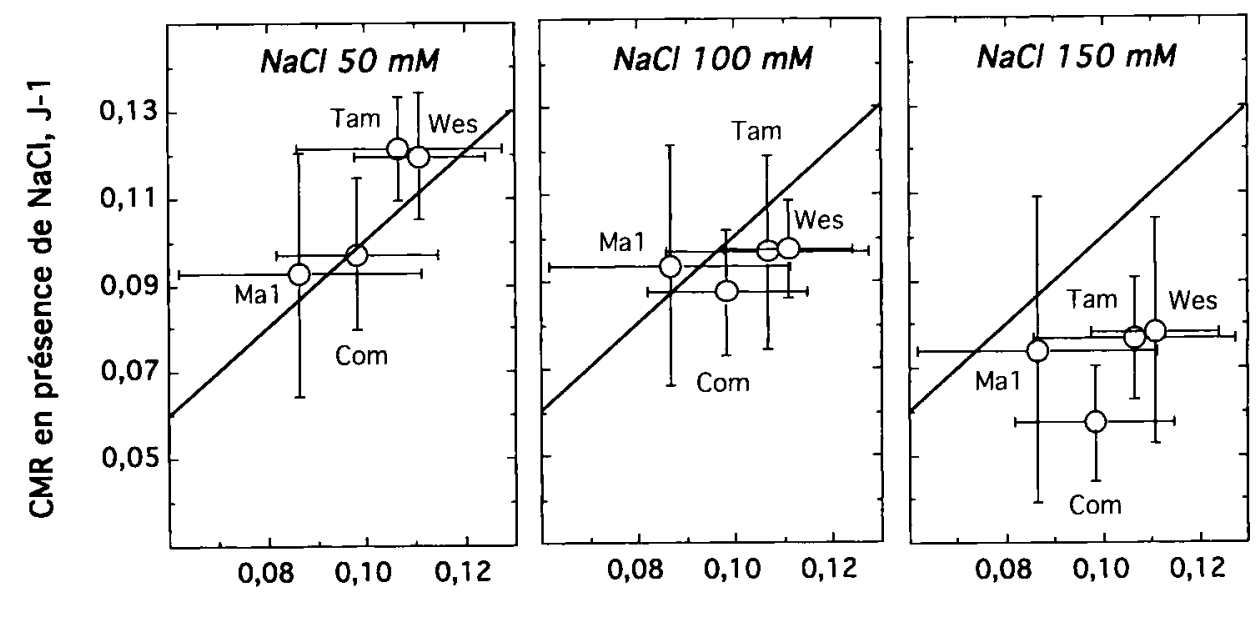

CMR en absence de $\mathrm{NaCl}, \mathrm{J}-1$
Fig 1. Effet de $\mathrm{NaCl}$ sur la production de matière sèche des quatre populations de lentille. Les graphiques mettent en relation la vitesse de croissance moyenne relative (CMR, jour $\left.{ }^{-1}\right)$ sur milieu témoin sans sel et sur milieu additionné de $\mathrm{NaCl}$. Moyennes de 16 plantes pour Com et 8 pour les autres, et intervalles de sécurité au seuil 0,05 . Masses initiales (mg MS. plante $\left.{ }^{-1}\right): 59 \pm 4$ (Com) ; $46 \pm 5$ (Tam) ; $54 \pm 4$ (Wes) ; $76 \pm 16$ (Mat). 
Fig 2. Évolution de la matière sèche de la plante entière (PE) chez les quatre populations de lentille cultivées en pots. Valeurs moyennes de 6 à 12 répétitions, intervalles de sécurité au seuil de 0,05.
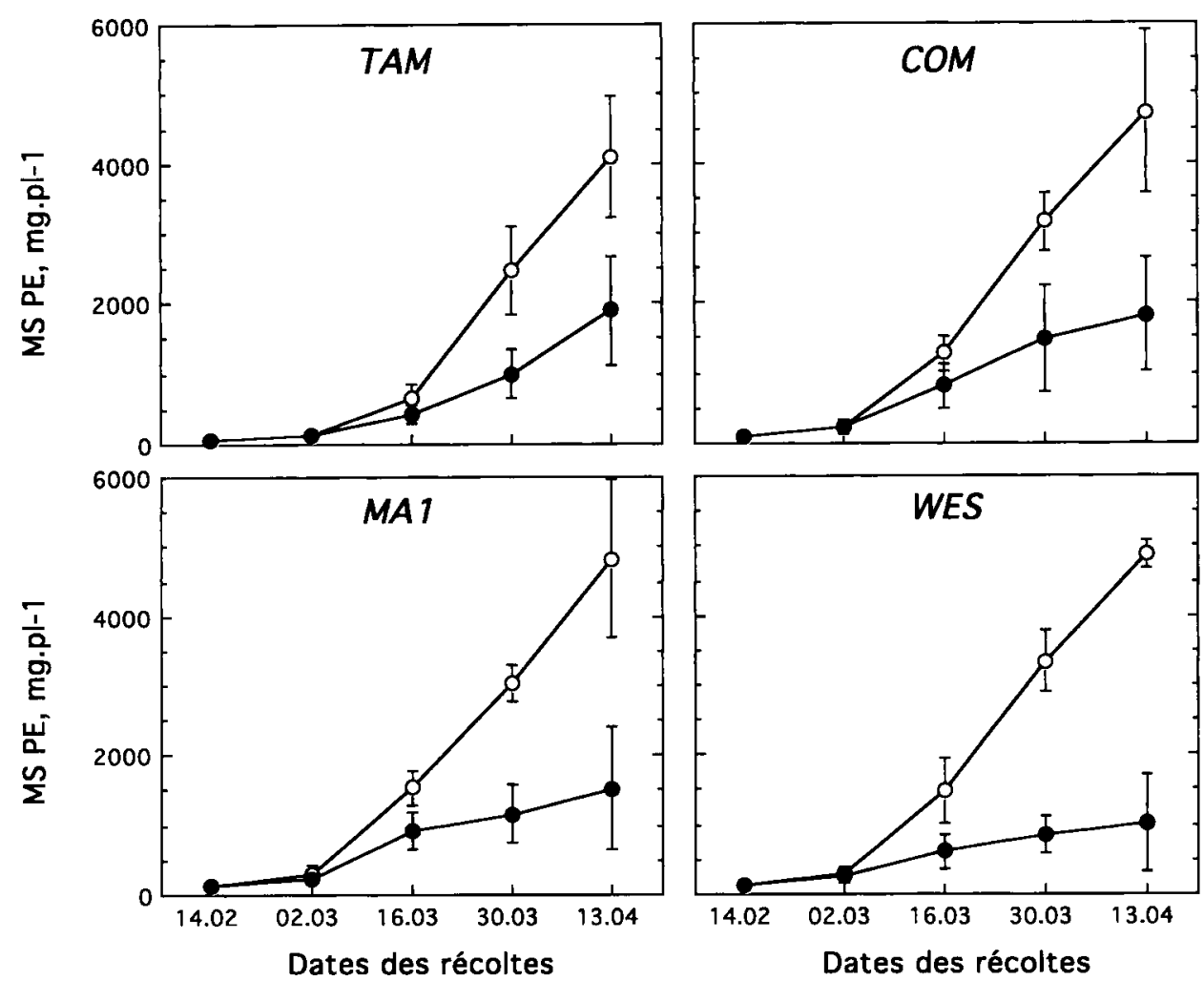

$\longrightarrow-\mathrm{NaCl} 10 \mathrm{mM}$

$\longrightarrow \mathrm{NaCl} 36 \mathrm{mM}$ dessèchement. Cet effet est moins marqué chez Tam et Com (les plus tolérantes du point de vue de la production de biomasse) que chez les deux autres populations. Wes (la plus sensible) est la plus affectée par le dessèchement des feuilles.

La culture en présence de $\mathrm{NaCl} 36 \mathrm{mM}$ entraîne un enrichissement en $\mathrm{Na}^{+}$important des feuilles jeunes (feuilles supérieures) (fig 5), qui est associé à une nécrose localisée, puis au dessèchement général du limbe, quand la teneur en sodium excède environ $2 \mathrm{mmol} . \mathrm{g}^{-1} \mathrm{MS}$. Selon la vitesse d'accumulation de $\mathrm{Na}^{+}$, le stade critique est atteint plus ou moins vite. Le blocage de la croissance foliaire intervient donc pour des stades de développement du feuillage plus ou moins avancés selon les populations. Chez Tam, le seuil critique de nécrose est atteint si tardivement que la production de MS des feuilles n'est pas affectée par le sel (par rapport au témoin). Chez Wes, au contraire, l'enrichissement rapide des feuilles en $\mathrm{Na}^{+}$fait que le stade nécrotique apparaît alors que la biomasse des feuilles sur milieu salé n'a atteint qu'environ la moitié de la valeur sur milieu témoin. Cette relation entre la nécrose foliaire et l'atteinte d'un seuil critique d'accumulation de $\mathrm{Na}^{+}$(et $\mathrm{Cl}^{-}$) est confirmée par la figure 7 qui compare les teneurs ioniques des feuilles médianes saines (vertes) et nécrosées, prélevées au même moment, immédiatement après la détection des premières nécroses. Comme chez les Citrus cultivés en présence de $\mathrm{NaCl}$ (Zid et Grignon, 1985), la nécrose se traduit par une élévation importante du rapport $\mathrm{Ca}^{2+} / \mathrm{K}^{+}$ (fig 7). Ce phénomène révèle probablement une perte de $\mathrm{K}^{+}$, cation cytoplasmique majeur, et une accumulation de $\mathrm{Ca}^{2+}$ (Cramer et al, 1985), cation normalement extracellulaire, dans les cellules mortes (Zid et Grignon, 1985). En outre, on voit que les feuilles vertes sur milieu contenant $\mathrm{NaCl} 36 \mathrm{mM}$ sont presque au niveau critique d'accumulation de $\mathrm{Na}^{+}$et $\mathrm{Cl}^{-}$, mais ne présentent pas encore le rapport $\mathrm{Ca}^{2+} / \mathrm{K}^{+}$caractéristique de l'accident nécrotique. Cependant, la population Tam, la plus tolérante, maintient des teneurs en $\mathrm{Ca}^{2+}$ et $\mathrm{K}^{+}$relativement plus élevées que Wes, dans ses feuilles vertes et à un degré moindre dans ses feuilles nécrosées. Le rôle de $\mathrm{Ca}^{2+}$ dans l'amélioration de la croissance en milieu salé a été souligné par Kent et Läuchli (1985) et plus récemment par Cachorro et al (1994).

\section{DISCUSSION ET CONCLUSION}

D'après la littérature (Das et Mehrotra, 1971 ; Ayoub, 1975 ; 1977) la lentille est modérément 

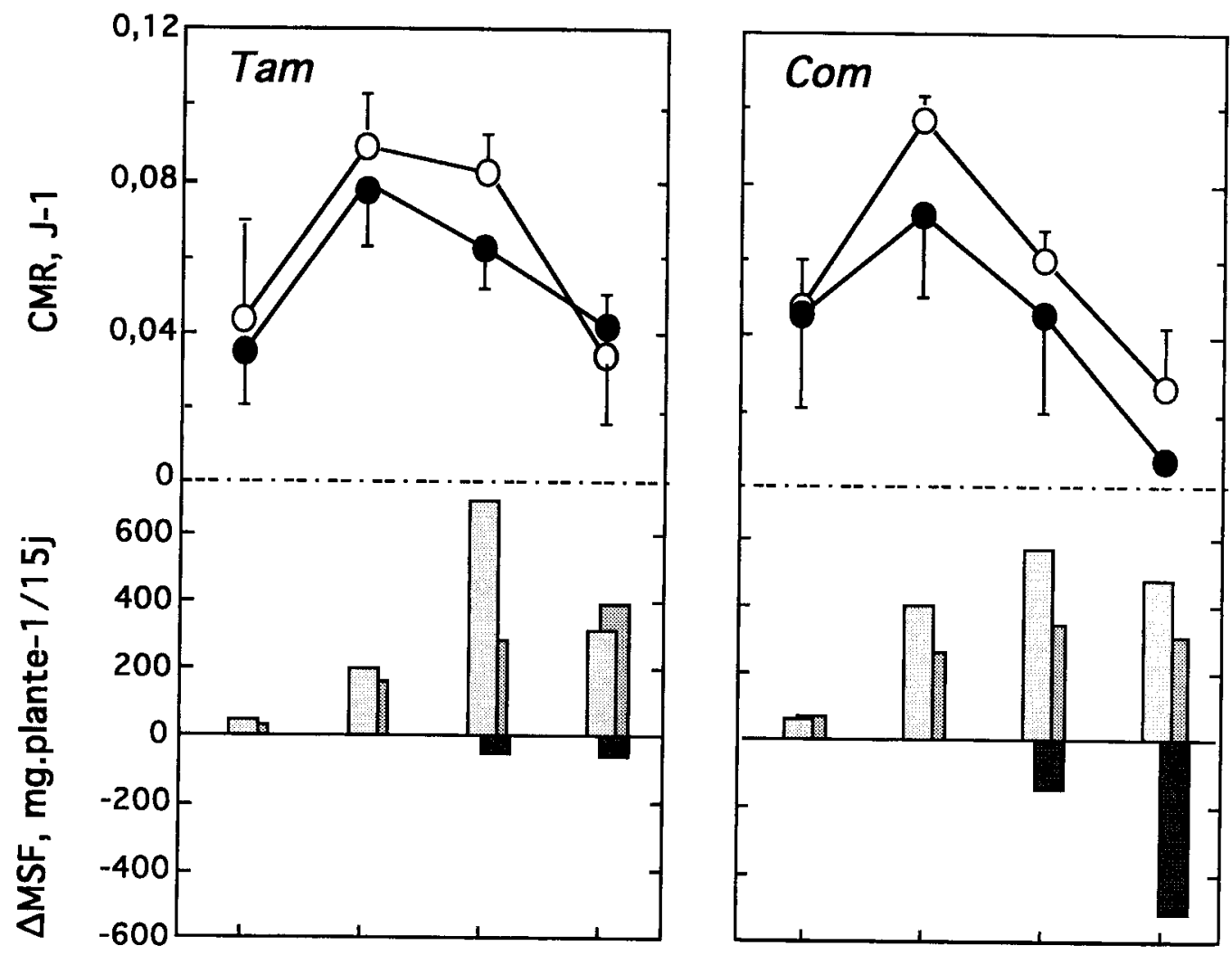

Fig 3. Croissance et production de feuilles par plante chez les quatre populations de lentille. Moyennes de 6 à 12 plantes et intervalles de sécurité au seuil de 0,05 .

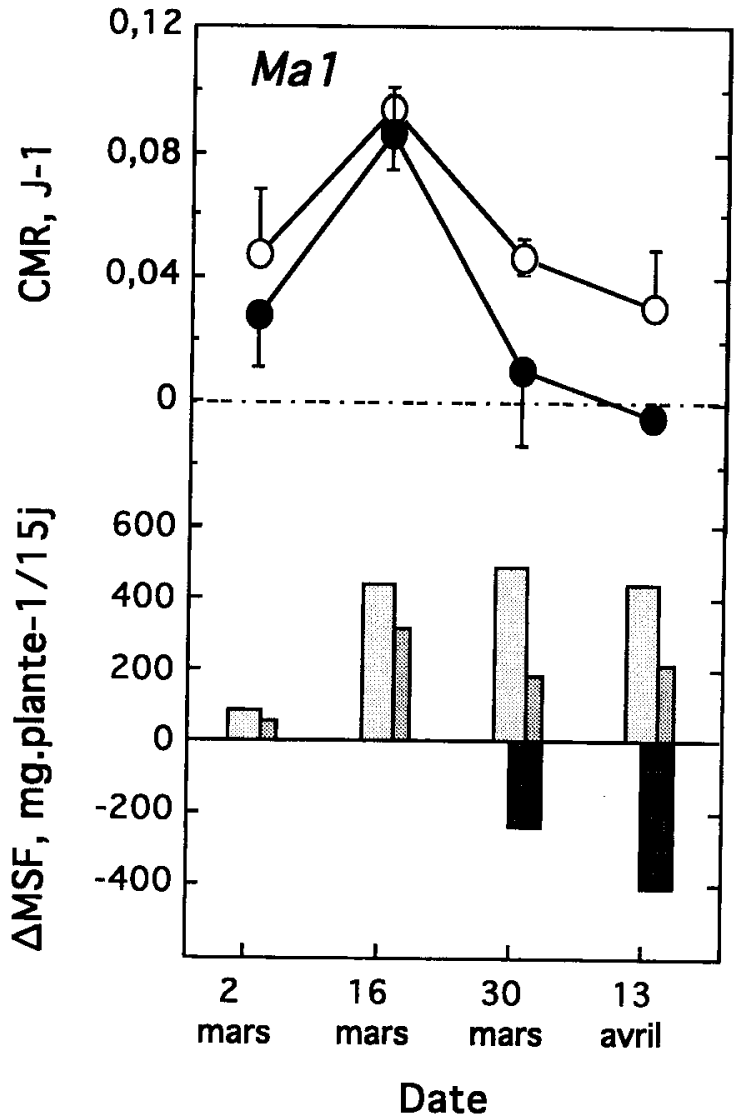

$\mathrm{O} \mathrm{NaCl} 10 \mathrm{mM}$

- $\mathrm{NaCl} 36 \mathrm{mM}$

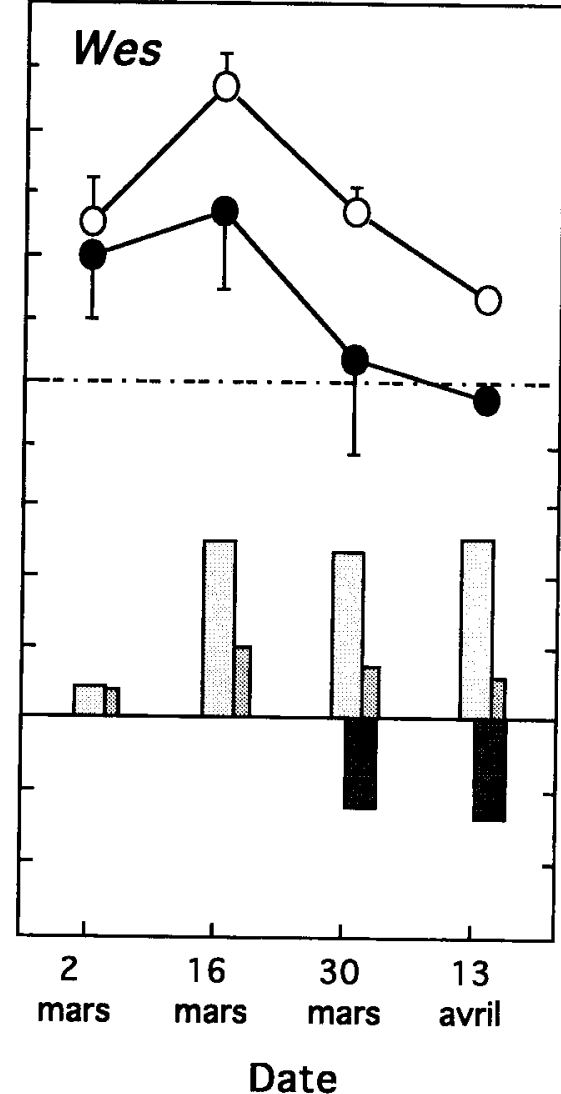

$\mathrm{NaCl} 10 \mathrm{mM}$ F. vertes $\mathrm{NaCl} 36 \mathrm{mM}$ F. vertes $\mathrm{NaCl} 36$ mM F. dess. 


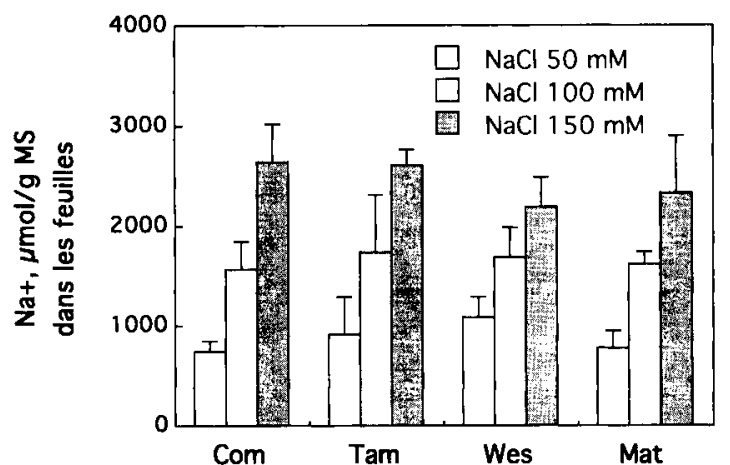

Fig 4. Effet de $\mathrm{NaCl}$ sur l'accumulation de $\mathrm{Na}^{+}$dans les feuilles des quatre populations de lentille. Dans les traitements témoins (sans $\mathrm{NaCl}$ ), les teneurs en $\mathrm{Na}^{+}$sont inférieures à $10 \mu$ éq. $\mathrm{g}^{-1} \mathrm{MS}$. Moyennes de 16 plantes pour Com et 8 pour les autres, et intervalles de sécurité au seuil 0,05.

tolérante à la salinité au stade germination mais plus sensible aux stades ultérieurs de la croissance. La tolérance au stade plantule, dans les conditions de nos expériences, ne reflète pas celle au stade adulte. $\mathrm{NaCl}$ exerce un effet inhibiteur sur la croissance des jeunes plantes (figs 1 et 3), mais cet effet ne paraît pas déterminer directement la production de biomasse aux stades avancés (floraison). À ces stades, la croissance paraît déterminée par l'élimination d'une partie des feuilles suite à une accumulation excessive de $\mathrm{Na}^{+}$et de $\mathrm{Cl}^{-}$. Le sel ne change pas le schéma morphogénétique des plantes puisque le nombre de feuilles des plantes traitées est voisin de celui des témoins (fig 6). En revanche, il agit sur la croissance en diminuant la biomasse de ces organes et en éliminant les feuilles qui atteignent le seuil d'accumulation toxique de $\mathrm{Na}^{+}$. Des résultats similaires obtenus chez le riz montrent que la survie des plantes est inversement corrélée aux teneurs de $\mathrm{Na}^{+}$dans les feuilles, quoique cette corrélation ne rende compte que de $30 \%$ de la variabilité variétale de la sensibilité au sel de cette espèce (Yeo et Flowers, 1989). Enfin, une analyse électrophysiologique récente (Chowdhury et al, 1995) montre que les variétés de riz les plus résistantes à la salinité sont celles qui excluent le plus efficacement $\mathrm{Na}^{+}$des cellules de leurs feuilles. Chez la lentille, cette toxicité du $\mathrm{Na}^{+}$accumulé est associée à des rapports $\mathrm{Ca}^{2+} / \mathrm{K}^{+}$très élevés (fig 7 ), caractéristiques de l'état de sénescence foliaire. $\mathrm{Ce}$ trait appuie I'hypothèse de Yeo et Flowers (1986) selon laquelle le sel accélère la sénescence, réduisant la durée de vie des feuilles. Cependant, comme pour le seuil de $\mathrm{Na}^{+}$dans les tissus, il n'y a pas de différences variétales
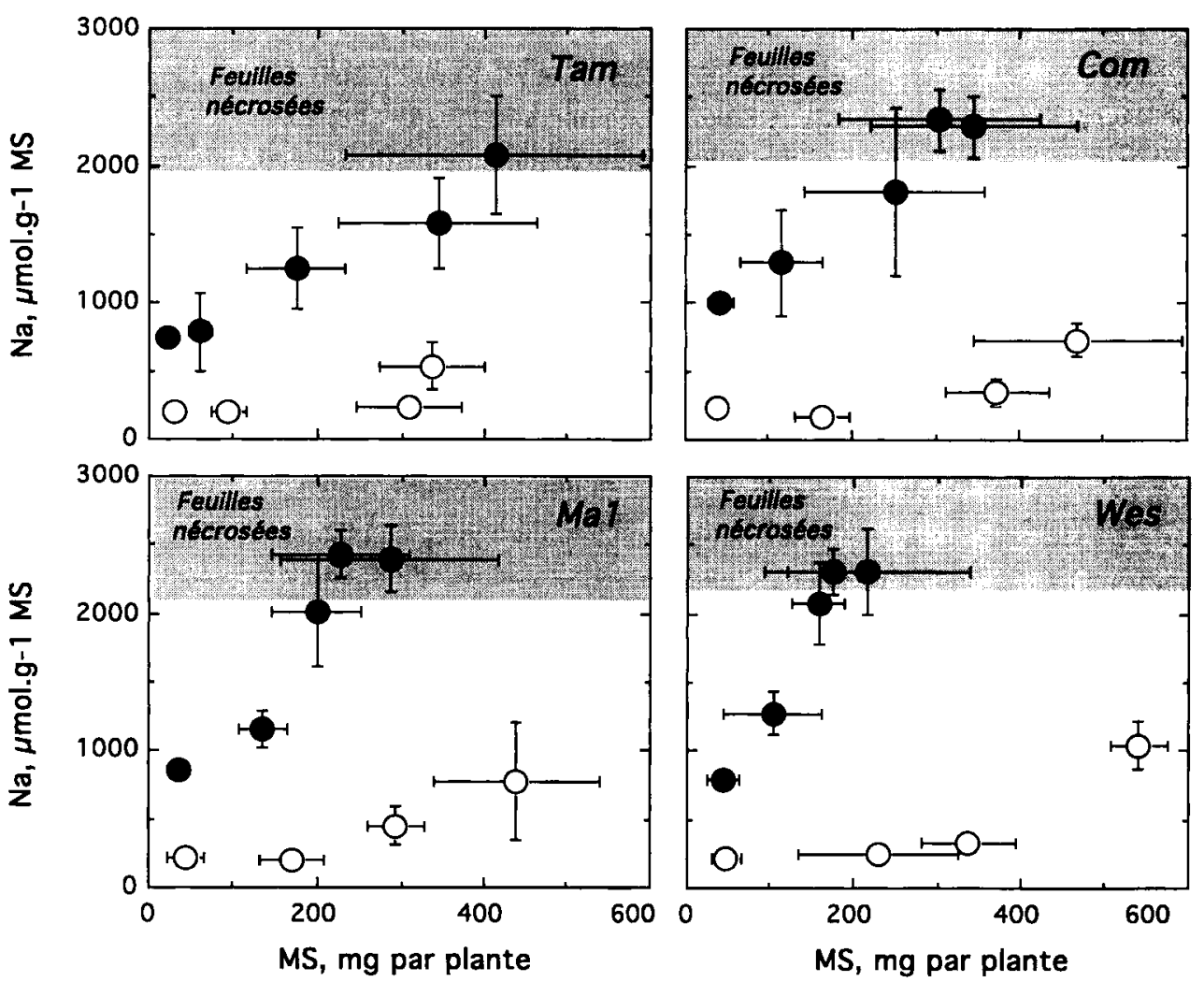

Fig 5. Teneurs en $\mathrm{Na}^{+}$des feuilles supérieures (correspondant à la touffe apicale des tiges) en relation avec leurs masses de matière sèche chez les populations de lentille. Valeurs moyennes de 6 à 12 répétitions, intervalles de sécurité au seuil de 0,05 . Étant donné que la biomasse des feuilles croît avec l'âge des plantes, pour chaque plante et chaque traitement les points successifs de gauche à droite correspondent à des âges croissants. 

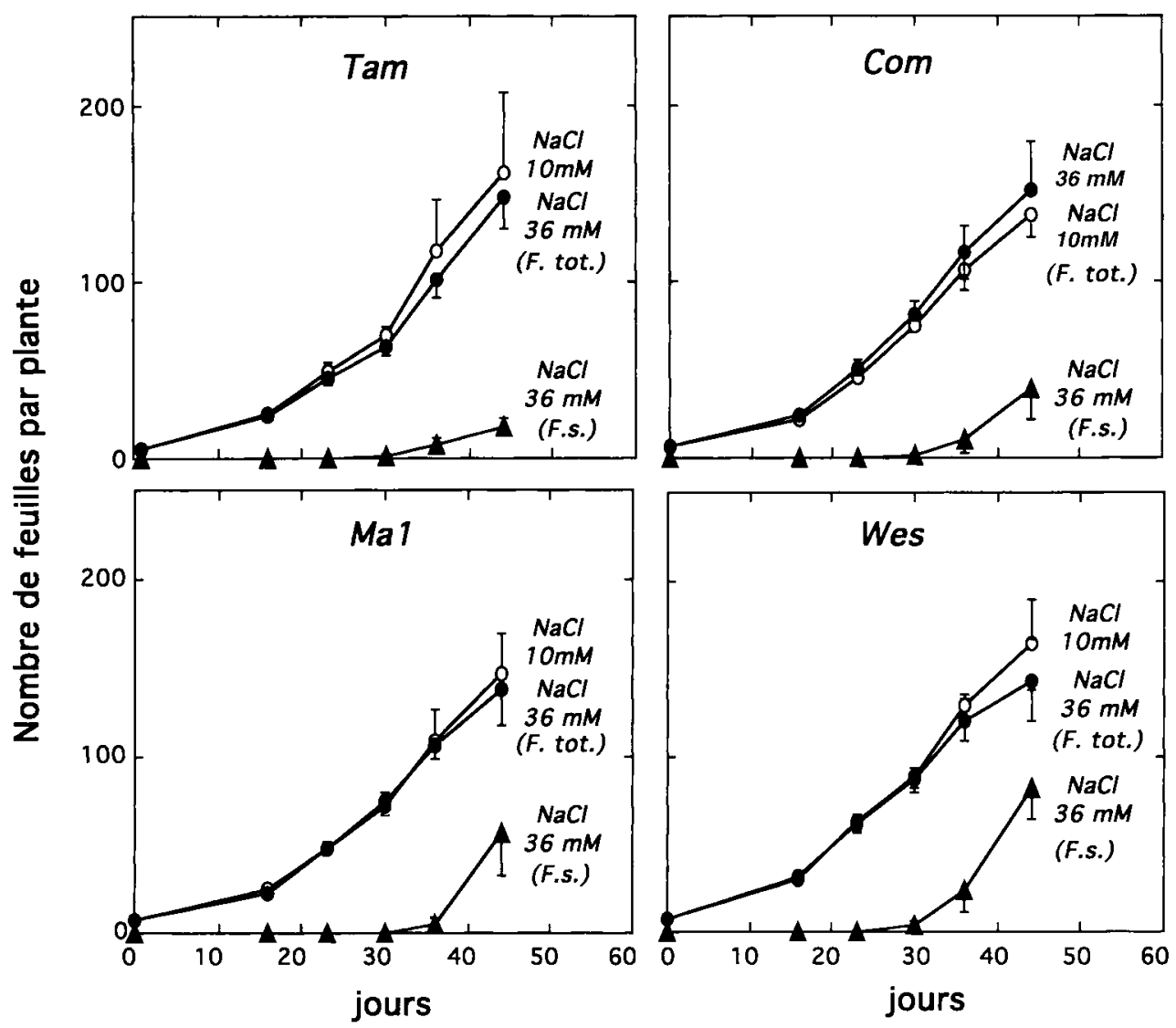

Fig 6. Évolution du nombre de feuilles par plante chez les quatre populations de lentille, cultivées sur milieux contenant $\mathrm{NaCl} 10 \mathrm{mM}$ et $36 \mathrm{mM}$. Intervalle de sécurité au seuil de 0,05 . F tot: nombre total de feuilles ; Fs : feuilles sèches.

Tableau III. Évolution de la matière sèche de la feuille chez les quatre populations de lentille cultivées en pots irrigués avec des solutions contenant $\mathrm{NaCl} 10 \mathrm{mM}$ et $36 \mathrm{mM}$. Masses moyennes par feuille saine (mg), calculées à partir des biomasses et des nombres de feuilles par plante.

Populations

$\mathrm{NaCl}(\mathrm{mM})$
Dates des récoltes

2 mars $\quad 16$ mars $\quad 30$ mars
2,7
2,2
4,9
4,3
5,3
4,3
4,0
3,5

3.9

3,3

6,6

4,7

7,9

5,6

7,1

3,8
6,7 nettes du rapport $\mathrm{Ca}^{2+} / \mathrm{K}^{+}$permettant d'expliquer la variabilité de la sensibilité au sel entre les quatre populations testées. Nos résultats indiquent que cette variabilité est plutôt due à une différence dans la capacité de maintenir assez de feuilles saines pour permettre la croissance de la plante en présence de sel. Cette capacité dépend de l'écart entre la vitesse d'élimination des feuilles par accumulation excessive de $\mathrm{Na}^{+}$ et la vitesse de production de nouvelles feuilles.
Lorsque le taux de mortalité foliaire excède celui de la production de nouvelles feuilles, la surface foliaire encore saine ne produit probablement pas assez d'assimilats pour alimenter les régions en croissance et le système racinaire, ce qui entraîne l'arrêt de croissance suivi de la mort des plantes (Munns et Termaat, 1986). La protection offerte par la croissance rapide (effet de dilution par la croissance du $\mathrm{Na}^{+}$accumulé dans les tissus) explique la relation entre vigueur de crois- 

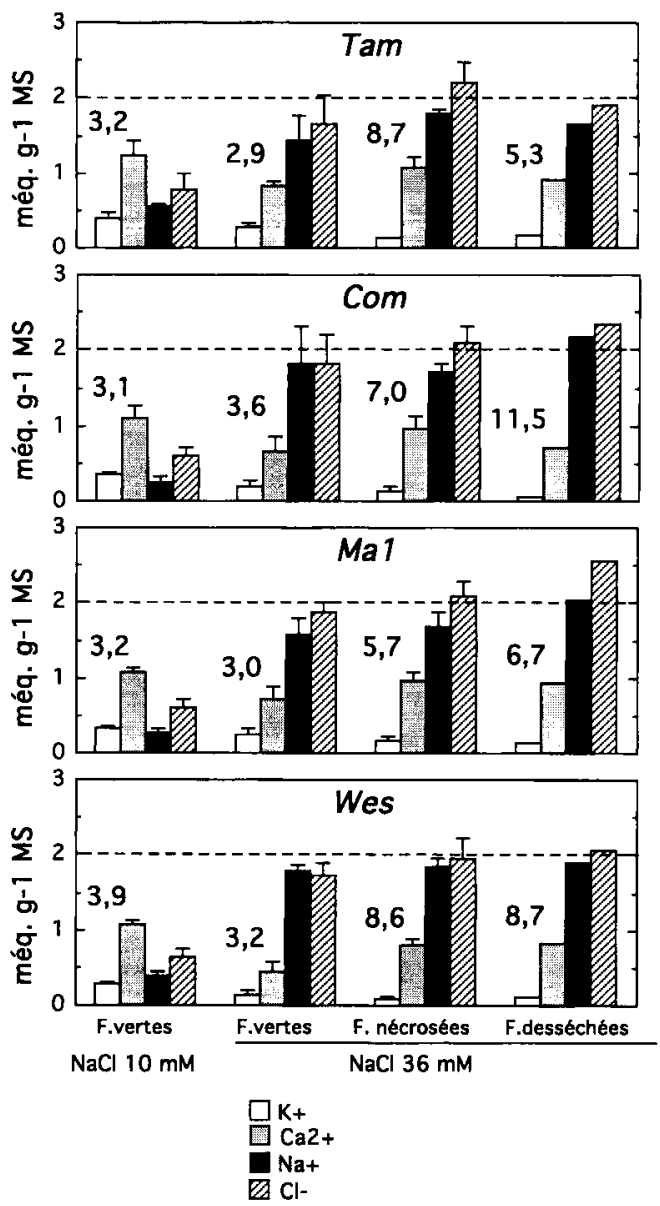

Fig 7. Accumulations ioniques dans les feuilles médianes après un mois de traitement sur milieux contenant $\mathrm{NaCl}$ $10 \mathrm{mM}$ et $36 \mathrm{mM}$. Moyennes de six répétitions, intervalles de sécurité au seuil de 0,05 . La ligne horizontale en tirets indique le seuil d'accumulation de $\mathrm{Na}^{+}$au-dessus duquel apparaissent les nécroses. Les nombres associés aux histogrammes correspondent aux valeurs du rapport $\mathrm{Ca}^{2+} / \mathrm{K}^{+}$.

sance et tolérance au sel signalée par plusieurs auteurs (Sarin, 1961 ; Greenway, 1965 ; Yeo et Flowers, 1984). Les variétés à croissance foliaire rapide sont capables de : i) retarder l'accès au seuil critique d'accumulation de $\mathrm{Na}^{+}$; et ii) maintenir un potentiel photosynthétique en produisant de nouvelles feuilles plus rapidement que le sel n'en élimine.

\section{RÉFÉRENCES}

Abel GH, Mackenzie AJ (1964) Salt tolerance of soybean varieties (Glycine max $L$ ) during germination and later growth. Crop Sci 4, 157-161

Ashraf M, Waheed A (1990) Screening of local/exotic accessions of lentil (Lens culinaris Medic) for salt tolerance at two growth stages. Plant Soil 128, 167176

Ashraf M, Mc Neilly T, Bradshow AD (1986a) The response of selected salt-tolerant and normal lines of four grass species to $\mathrm{NaCl}$ in sand culture. New Phytol 104, 453-461

Ashraf M, Mc Neilly T, Bradshaw AD (1986b) The response to $\mathrm{NaCl}$ and ionic content of selected salttolerant and normal lines of three legume forage species in sand culture. New Phytol 104, 463-471

Ayoub AT (1975) Studies on the incidence of the heavy lentil and chick-peas plants losses. Annual Report, Hudeiba Agricultural Research Station, EdDamer, Sudan

Aoub AT (1977) Salt tolerance of lentil (Lens esculenta L). J Hort Sci 52, 163-168

Blum A (1985) Breeding crop varieties for stress environments. CRC Critical Reviews in Plant Sciences 2, 199-238

Cachorro P, Ortiz A, Cerda A (1994) Implications of calcium nutrition on the response of Phaseolus vulgaris L to salinity. Plant Soil 159, 205-212

Chapman HD (1968) The mineral nutrition of Citrus. In : The Citrus Industry. II. (W Reuther, LD Batchelor, HD Webber, eds), Univ California Press, Berkeley, 127-289

Chowdhury MAM, Moseki B, Bowling DJF (1995) A method for screening rice plants for salt tolerance. Plant Soil 171, 317-322

Cramer GR, Laüchli A, Polito VS (1985) Displacement of $\mathrm{Ca}^{2+}$ by $\mathrm{Na}^{+}$from the plasmalemma of root cells. A primary response to salt stress? Plant Physiol 79, 207-211

Das SK, Mehrotra CL (1971) Salt tolerance of some agricultural crops during early growth stages. Indian J Agric Sci 41, 882-888

El Gibaly H, Goumah H (1969) The effect of salinization on the growth and yield of sugar cane. Beitr Trop Subtrop Laudwirt Tropenveterinaermed 7, 2739

Epstein E (1983) Crops tolerant of salinity and other mineral stresses. In : Better Crops for Food. Ciba Found Symp 97, Pitman Books, Londres, 61-82

Epstein E, Norlyn JD, Rush DW, Kingsbury RW, Kelley DB, Cunningham GA, Wrona AF (1980) Saline culture of crops: a genetic approach. Science 210, 399-404

Greenway H (1965) Plant responses to saline substrates. VII. Growth and ion uptake throughout plant development in two varieties of Hordeum vulgare $\mathrm{L}$. Aust J Biol Sci 18, 763-779

Greenway H, Munns R (1980) Mechanisms of salt tolerance in nonhalophytes. Annu Rev Plant Physio/ 31, $149-190$

Hachicha M, Job JO, Mtimet A (1994) Les sols salés et la salinisation en Tunisie. Sols de Tunisie 15, 271341

Hewitt EJ (1966) Sand and water culture methods used in the study of plant nutrition. 2nd ed, Commonwealth Bureau of Horticulture Tech Comm, 22

Hunt OJ (1965) Salt tolerance in intermediate wheatgrass (Agropyron intermedium). Crop Sci 5, 407409 
Jana MK, Slinkard AE (1979) Screening for salt tolerance in lentils. Lens $6,25-27$

Katerji N, Van Hoorn JW, Hamdy A, Karam F (1994) Effect of salinity on emergence and on water stress and early seedling growth of sunflower and maize. Agri Water Manage 26, 81-91

Kent L, Läuchli A (1985) Germination and seedling growth of cotton; salinity-calcium interactions. Plant Cell Environ 8, 155-159

Kingsbury RW, Epstein E (1984) Selection for saltresistant spring wheat. Crop Sci 24, 310-315

Kuiper PJC, Kuiper D, Schiut J (1988) Root functioning under stress conditions: an introduction. Plant Soil 111, 249-253

Lehman WF, Rutger JN, JN Robinson FE, Kaddah M (1984) Value of rice characteristics in selection for resistance to salinity in arid environment. Agron $J$ $76,366-370$

Maas EV, Hoffman GJ (1977) Crop salt tolerance-current assessment. J Irrig Drainage Div, ASCE 103 (IR2), 115-134

Munns R, Termaat A (1986) Whole plant responses to salinity. Aust J Plant Physiol 13, 143-160

Norlyn JB (1980) Breeding salt-tolerant crop plants. In: Genetic Engineering of Osmoregulation: Impact on Plant Productivity for Food, Chimicals and Energy (DW Rains, RC Valentine, A Hollaender, eds) Plenum Press, New York, 293-309

Norlyn JB, Epstein E (1984) Variability in salt tolerance of four triticale lines at germination and emergence. Crop Sci 24, 1090-1092

Ream CL, Furr JR (1976) Salt tolerance of some Citrus species, relatives and hybrids tested as rootstocks. J Am Soc Hortic Sci 101, 265-267
Sarin MN (1961) Physiological studies on salt tolerance in crop plants. XII. Influence of sodium sulphate on early seedling growth of wheat and grain varieties. Agr Univ J Res Sci 10, 41-60

Shannon MC (1979) In quest of rapid screening techniques for plant salt tolerance. Hortic Sci 14, 587589

Shannon MC, McCreight JD, Draper JH (1983) Screening tests for salt tolerance in lettuce. $J \mathrm{Am}$ Soc Hortic Sci 108, 225-230

Venus JC, Causton DR (1979) Plant growth analysis: a reexamination of the methods of calculation of relative growth and net assimilation rates without using fitted functions. Ann Bot 43, 633-638

Yeo AR, Flowers TJ (1984) Mechanism of salinity resistance in rice and their as physiological criteria in plant breeding. In : Salinity Tolerance in Plant Strategies for Crop Improvement (RC Staples, GA Toennienssen, eds), Wiley \& Sons, New York, 151170

Yeo AR, Flowers TJ (1986) Salinity resistance in rice (Oryza sativa $L$ ) and pyramiding approach to breeding varieties for saline soils. Aust $J$ Plant Physiol 13, 163-173

Yeo AR, Flowers TJ (1989) Selection for physiological characters: examples from breeding for salt tolerance. In: Plants Under Stress (GJ Hamlyn, TJ Flowers, MB Jones, eds), Cambridge University Press, Cambridge, 217-234

Zid E (1989) Étude de la nécrose foliaire chez différentes espèces de Citrus cultivées en présence de $\mathrm{NaCl}$. Rev Fac Sci Tunis 4, 145-160

Zid E, Grignon C (1985) Sodium-calcium interactions in leaves of Citrus aurantium $\mathrm{L}$ grown in the presence of NaCl. Physiol Vég 23, 895-903 